\title{
The X-ray outburst of the Galactic Centre magnetar as monitored by Chandra and XMM-Newton
}

\author{
Francesco Coti Zelati ${ }^{* 1,2,3}$ Nanda Rea, ${ }^{2.4}$ Alessandro Papitto, ${ }^{4}$ Daniele Viganò, ${ }^{4}$ \\ José A. Pons, ${ }^{5}$ Roberto Turolla, ${ }^{6,7}$ Paolo Esposito,,${ }^{8,9}$ Daryl Haggard, ${ }^{10}$ Frederick \\ K. Baganoff, ${ }^{11}$ Gabriele Ponti, ${ }^{12}$ Gianluca Israel, ${ }^{13}$ Sergio Campana, ${ }^{3}$ Diego \\ F. Torres, ${ }^{4,14}$ Andrea Tiengo, ${ }^{8,15,16}$ Sandro Mereghetti, ${ }^{8}$ Rosalba Perna, ${ }^{17}$ Silvia \\ Zane, ${ }^{7}$ Roberto P. Mignani, ${ }^{8,18}$ Andrea Possenti, ${ }^{20}$ Luigi Stella $^{13}$ \\ ${ }^{1}$ Università dell'Insubria, via Valleggio 11, I-22100 Como, Italy \\ 2 Anton Pannekoek Institute for Astronomy, University of Amsterdam, Postbus 94249, \\ NL-1090-GE Amsterdam, The Netherlands \\ ${ }^{3}$ INAF - Osservatorio Astronomico di Brera, via Bianchi 46, I-23807 Merate (LC), Italy \\ ${ }^{4}$ Institute of Space Sciences (CSIC-IEEC), Faculty of Science, Campus UAB, Torre C5-parell, 2 a \\ planta, E-08193 Bellaterra, Spain \\ ${ }^{5}$ Departament de Fisica Aplicada, Universitat d'Alacant, Ap. Correus 99, E-03080 Alacant, \\ Spain \\ ${ }^{6}$ Dipartimento di Fisica e Astronomia, Università di Padova, via F. Marzolo 8, I-35131 Padova, \\ Italy \\ ${ }^{7}$ Mullard Space Science Laboratory, University College London, Holmbury St. Mary, Dorking, \\ Surrey RH5 6NT, UK \\ ${ }^{8}$ INAF - Istituto di Astrofisica Spaziale e Fisica Cosmica, via E. Bassini 15, I-20133 Milano, Italy \\ ${ }^{9}$ Harvard-Smithsonian Center for Astrophysics, 60 Garden Street, Cambridge, MA 02138, USA \\ ${ }^{10}$ Department of Physics and Astronomy, Amherst College, Amherst, MA 01002-5000, USA \\ ${ }^{11}$ Kavli Institute for Astrophysics and Space Research, Massachusetts Institute of Technology, \\ Cambridge, MA 02139, USA \\ ${ }^{12}$ Max Planck Institute fur Extraterrestriche Physik, Giessenbachstrasse, D-85748 Garching, \\ Germany \\ ${ }^{13}$ INAF - Osservatorio Astronomico di Roma, via Frascati 33, I-00040 Monteporzio Catone, \\ Roma, Italy \\ ${ }^{14}$ Institució Catalana de Recerca i Estudis Avançats (ICREA), E-08010 Barcelona, Spain \\ 15 Istituto Universitario di Studi Superiori, piazza della Vittoria 15, I-27100 Pavia, Italy \\ ${ }^{16}$ Istituto Nazionale di Fisica Nucleare, Sezione di Pavia, via A. Bassi 6, I-27100 Pavia, Italy \\ ${ }^{17}$ Department of Physics and Astronomy, Stony Brook University, Stony Brook, NY 11794, USA \\ ${ }^{18}$ Kepler Institute of Astronomy, University of Zielona Gora, Lubuska 2, 65-265, Zielona Góra, \\ Poland \\ ${ }^{19}$ INAF - Osservatorio Astronomico di Cagliari, via della Scienza 5, 09047, Selargius, Italy \\ E-mail: francesco.cotizelatiabrera.inaf.it
}


In 2013 April a new magnetar, SGR 1745-2900, was discovered as it entered an outburst, at an angular separation of only 2.4 arcsec from the supermassive black hole at the centre of the Milky Way, Sagittarius A*. The new source was detected both in the radio and X-ray bands, with a peak X-ray luminosity $L_{\mathrm{X}} \sim 5 \times 10^{35} \mathrm{erg} \mathrm{s}^{-1}$, and it has a spin-down magnetic field of $\sim$ $2 \times 10^{14} \mathrm{G}$. Here we report on the long-term Chandra (25 observations) and XMM-Newton (eight observations) X-ray monitoring campaign of SGR 1745-2900 from the onset of the outburst in 2013 April 2013 until 2014 September. This unprecedented data set allows us to refine the timing properties of the source, as well as to study the outburst spectral evolution as a function of time and rotational phase. Our timing analysis confirms the increase in the spin period derivative by a factor of $\sim 2$ around 2013 June, and reveals that a further increase occurred between 2013 October 30 and 2014 February 21 . We find that the period derivative changed from $6.6 \times 10^{-12}$ to $3.3 \times 10^{-11} \mathrm{~s} \mathrm{~s}^{-1}$ in $1.5 \mathrm{yr}$. On the other hand, this magnetar shows a slow flux decay compared to other magnetars and a rather inefficient surface cooling. In particular, starquake-induced crustal cooling models alone have difficulty in explaining the high luminosity of the source for the first $\sim 200 \mathrm{~d}$ of its outburst, and additional heating of the star surface from currents flowing in a twisted magnetic bundle is probably playing an important role in the outburst evolution.

Swift: 10 Years of Discovery

2-5 December 2014

La Sapienza University, Rome, Italy

${ }^{*}$ Speaker. 


\section{Introduction}

In the past decade, extensive study of magnetars in outburst has led to a number of unexpected discoveries which have changed our understanding of these objects ([四], [四]). The detection of typical magnetar-like bursts and a powerful enhancement of the persistent emission unveiled the existence of three low magnetic field $\left(B<4 \times 10^{13} \mathrm{G}\right)$ magnetars ([目], [焑], [可], [回]). Recently, an absorption line at a phase-variable energy was discovered in the X-ray spectrum of SGR 0418+5729. This, if interpreted in terms of a proton cyclotron feature, provides a direct estimate of the magnetic field strength close to the neutron star surface ([प]).

In 2013 April a new magnetar, SGR 1745-2900, was discovered as it entered an outburst at only 2.4 arcsec from the supermassive black hole at the Centre of the Milky Way, Sgr A* ([[]], [Q]], [ए]]). SGR 1745-2900 has a spin-down magnetic field of $\sim 2 \times 10^{14} \mathrm{G}$ and holds the record as the closest neutron star to a black hole detected to date. The angular separation of $2.4 \pm 0.3 \operatorname{arcsec}$ from $\mathrm{Sgr} \mathrm{A*}$ corresponds to a minimum physical separation of $0.09 \pm 0.02 \mathrm{pc}$ (at a $95 \%$ confidence level) for an assumed distance of $8.3 \mathrm{kpc}$. The source has been observed daily with Swift/XRT until 2014 October, and its 2-10 keV flux has decayed steadily during this time interval ([ए]]).

Here we report on the X-ray long-term monitoring campaign of SGR 1745-2900 covering the first $1.5 \mathrm{yr}$ of the outburst decay. In Section 2 we describe the Chandra and XMM-Newton observations and the data analysis. In Section 3 we discuss our results; conclusions follow in Section 4.

\section{Observations and data analysis}

Chandra observed SGR 1745-2900 twenty-six times between 2013 April 29 and 2014 August 30. The first observation was performed with the HRC to have the best spatial accuracy to localize the source in the crowded region of the Galactic Centre ([ए]]]). The remaining observations were performed with the ACIS in faint timed-exposure imaging mode with a $1 / 8$ sub-array (time resolution of $0.4 \mathrm{~s}$ ), and in three cases with the HETG. The source was positioned on the back-illuminated S3 chip. Eight observations were carried out by XMM-Newton/EPIC, with the pn CCD camera operated in full-frame window mode (time resolution of $73.4 \mathrm{~ms}$ ) in all cases.

\subsection{Timing analysis}

We derive a phase coherent solution (solution A, see Table $\mathbb{W}$ ) that is able to model the pulse phase evolution before a $115 \mathrm{~d}$ observations gap starting at MJD 56600, and which is compatible with the solution given by [ए2] for the partly overlapping interval MJD $56457-56519$. After the observation gap, solution A is no longer able to provide a good description of pulse phases, and we are only able to find a solution based on the analysis of the spin frequency evolution (solution B, see Table $\mathbb{~}$ ). We then use timing solution A (up to MJD 56594.1) and solution B (from MJD 56709.5 onwards) to fold all background-subtracted and exposure-corrected light curves at the neutron star spin period during the corresponding observation (see the left panel of Fig. $\mathrm{W}$ ). This allows us to extract the temporal evolution of the pulsed fraction, defined as PF $=[\operatorname{Max}-\operatorname{Min}] /[\operatorname{Max}+\operatorname{Min}]$ (Max and Min being the maximum and the minimum count rate of the pulse profile, respectively; see the right-hand panel of Fig. (W). 
Table 1: Timing solutions. Errors were evaluated at the $1 \sigma$ confidence level, scaling the uncertainties by the value of the rms $\left(\sqrt{\chi_{V}^{2}}\right)$ of the respective fit to account for the presence of unfitted residuals.

\begin{tabular}{|c|c|c|c|c|}
\hline Solution & Rea et al. (2013) [0] & Kaspi et al. (2014) [ए2] & This work (solution A) & This work (solution B) \\
\hline Epoch $T_{0}$ (MJD) & 56424.5509871 & 56513.0 & 56513.0 & 56710.0 \\
\hline Validity range (MJD) & $56411.6-56475.3$ & $56457-56519$ & $56500.1-56594.1$ & $56709.5-56929$ \\
\hline$P\left(T_{0}\right)(\mathrm{s})$ & $3.7635537(2)$ & $3.76363824(13)$ & $3.76363799(7)$ & $3.7639772(12)$ \\
\hline$\dot{P}\left(T_{0}\right)$ & $6.61(4) \times 10^{-12}$ & $1.385(15) \times 10^{-11}$ & $1.360(6) \times 10^{-11}$ & $3.27(7) \times 10^{-11}$ \\
\hline$\ddot{P}\left(\mathrm{~s}^{-1}\right)$ & $4(3) \times 10^{-19}$ & $3.9(6) \times 10^{-19}$ & $3.7(2) \times 10^{-19}$ & $(-1.8 \pm 0.8) \times 10^{-19}$ \\
\hline$v\left(T_{0}\right)(\mathrm{Hz})$ & $0.265706368(14)$ & $0.265700350(9)$ & $0.26570037(5)$ & $0.26567642(9)$ \\
\hline$\dot{v}\left(T_{0}\right)\left(\mathrm{Hz} \mathrm{s}^{-1}\right)$ & $-4.67(3) \times 10^{-13}$ & $-9.77(10) \times 10^{-13}$ & $-9.60(4) \times 10^{-13}$ & $-2.31(5) \times 10^{-12}$ \\
\hline$\ddot{v}\left(\mathrm{~Hz} \mathrm{~s}^{-2}\right)$ & $-3(2) \times 10^{-20}$ & $-2.7(4) \times 10^{-20}$ & $-2.6(1) \times 10^{-20}$ & $(1.3 \pm 0.6) \times 10^{-20}$ \\
\hline rms residual & $0.15 \mathrm{~s}$ & $51 \mathrm{~ms}$ & $0.396 \mathrm{~s}$ & $1.0 \mu \mathrm{Hz}$ \\
\hline$\chi_{v}^{2}$ (d.o.f.) & $0.85(5)$ & $1.27(41)$ & $6.14(44)$ & $0.66(10)$ \\
\hline
\end{tabular}
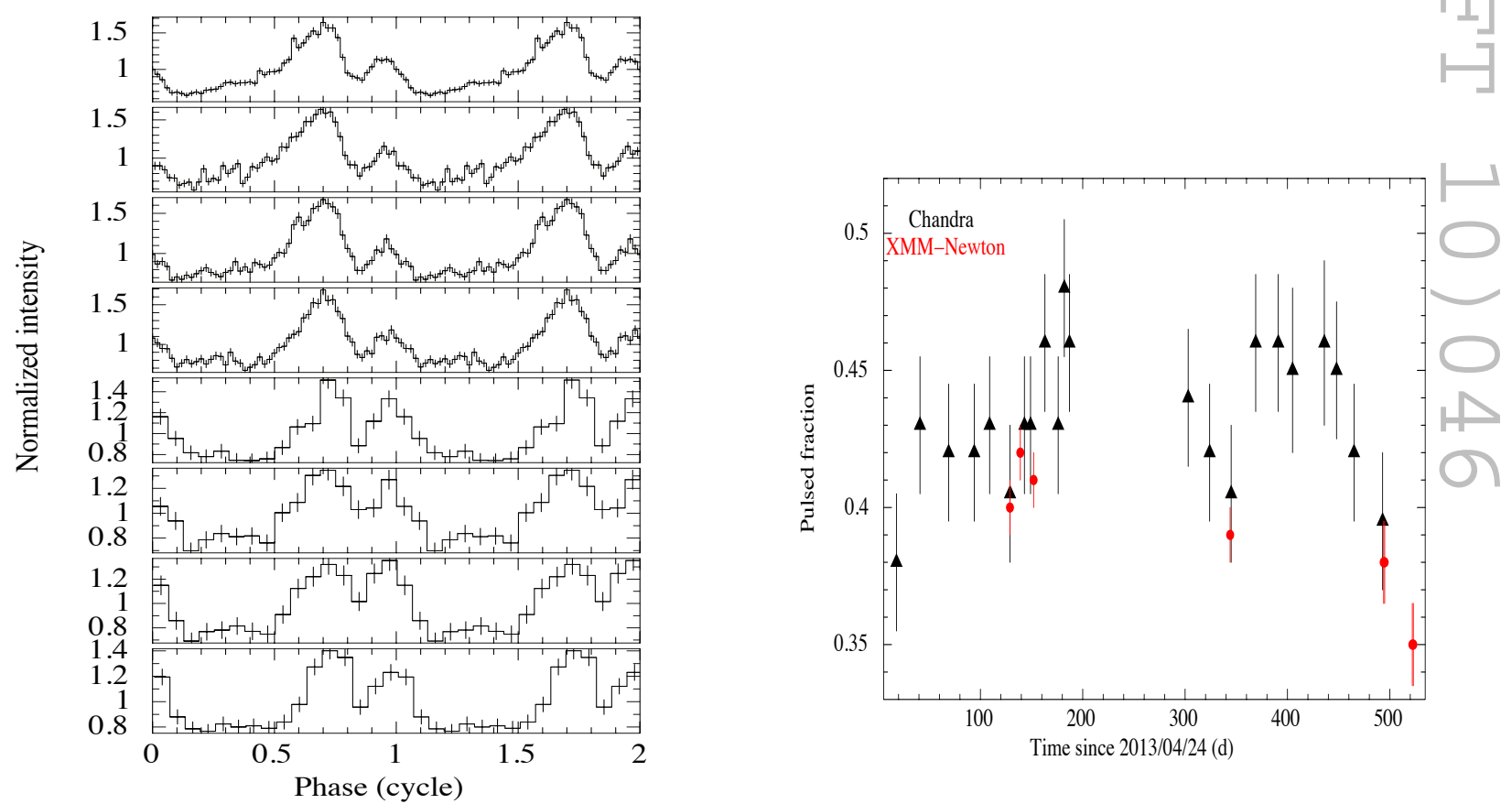

Figure 1: Left-hand panel: pulse profiles of SGR 1745-2900 in the 0.3-10 keV energy range obtained from XMM-Newton/EPIC-pn observations. Two cycles are shown for clarity. Right-hand panel: temporal evolution of the pulsed fraction (see text for our definition) in the $0.3-10 \mathrm{keV}$ band. Uncertainties on the values were obtained by propagating the errors on the maximum and minimum count rates. 


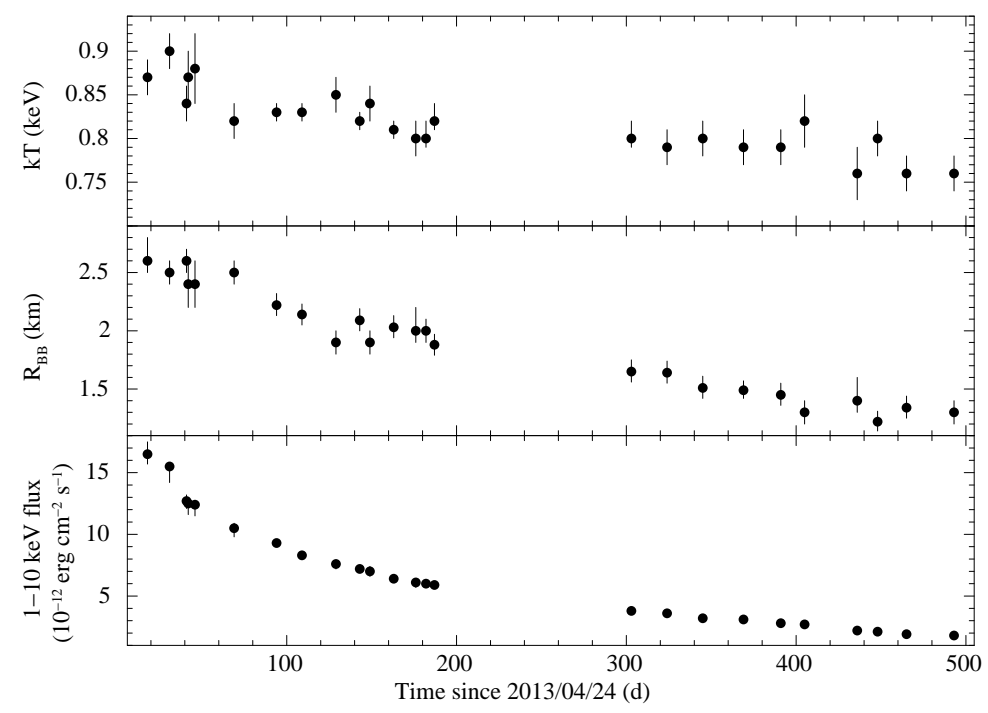

Figure 2: Temporal evolution of the spectral parameters for the blackbody model and of the absorbed intrinsic blackbody flux of SGR 1745-2900 in the 1-10 keV energy range from Chandra observations. The blackbody emitting radius is calculated assuming a source distance of $8.3 \mathrm{kpc}$. All errors are reported at a $90 \%$ confidence level for a single parameter of interest $\left(\Delta \chi^{2}=2.706\right)$.

\subsection{Spectral analysis}

A joint fit of all Chandra non-grating spectra with an absorbed blackbody model (in the 0.3-8 $\mathrm{keV}$ energy range, with the hydrogen column density tied to be the same between all the observations) yields $\chi_{v}^{2}=1.00$ for 2282 d.o.f., with $N_{\mathrm{H}}=1.90(2) \times 10^{23} \mathrm{~cm}^{-2}$ (at a $90 \%$ confidence level). When an absorbed power law model is used $\left(\chi_{v}^{2}=1.05\right.$ for 2282 d.o.f.), we obtain large values for the photon index $(\Gamma=4.2-4.9)$ and the photoelectric absorption $\left(N_{\mathrm{H}} \sim 3 \times 10^{23} \mathrm{~cm}^{-2}\right)$. These are likely an artifact of the fitting process which tends to increase the absorption to compensate the large flux at low energies defined by the power law. The addition of a second component to the blackbody (another blackbody or a power law), is not statistically required. A single absorbed blackbody thus provides the best modeling of the source spectrum in the $0.3-8 \mathrm{keV}$ energy range. Zeroth-order spectral data of the three grating observations were fitted together and independently with this model, fixing $N_{\mathrm{H}}$ to that obtained in non-grating fit (see Fig. [).

Based on the results of the Chandra analysis, we fit jointly all the XMM-Newton/EPIC-pn spectra in the $2-12 \mathrm{keV}$ band with an absorbed blackbody model. We obtain $\chi_{v}^{2}=2.2$ for 636 d.o.f., with large residuals at high energies. The latter disappear if an absorbed power law component is added $\left(\chi_{v}^{2}=1.13\right.$ for 624 d.o.f.; see the left-hand panel of Fig. [3). We also apply a 3D resonant cyclotron scattering model ([ए3]], [14]], [15]), obtaining $\chi_{v}^{2}=1.14$ for 624 d.o.f. (see the right-hand panel of Fig. [1). Both models successfully reproduce the soft X-ray part of the SGR 1745-2900 spectra up to $\sim 12 \mathrm{keV}$, implying that, similar to other magnetars, the reprocessing of the thermal emission by a dense, twisted magnetosphere produces a non-thermal component ([ए]]). The power law detected by XMM-Newton is consistent with that observed by NuSTAR ([ए2], and its very low contribution below $8 \mathrm{keV}$ is consistent with its non-detection in our Chandra data. 

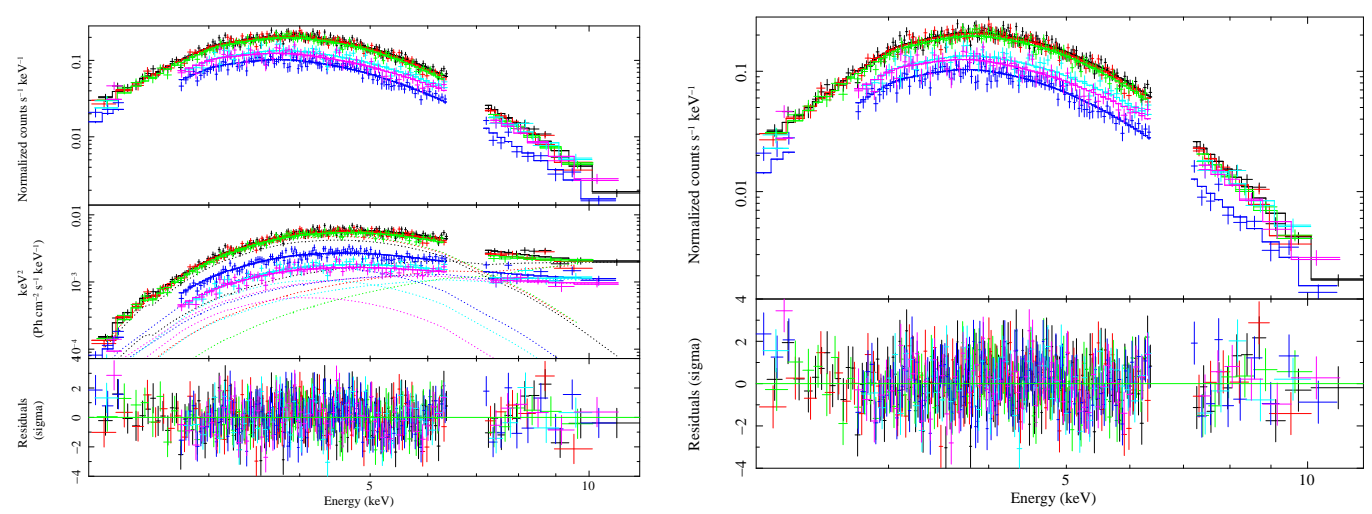

Figure 3: Results of the phase-averaged spectral analysis for the XMM-Newton/EPIC-pn observations of SGR 1745-2900. Spectra were fitted together in the 2-12 keV energy range and after removal of the Fe XXV and S XV lines from the supernova remnant Sgr A east ([ए]]). Left-hand panel: spectra fitted with an absorbed blackbody plus power law model. $E^{2} f(E)$ unfolded spectra together with the contributions of the two additive components and residuals (in units of standard deviations) are also shown. Right-hand panel: spectra fitted with an absorbed 3-D resonant cyclotron scattering model. Residuals (in units of standard deviations) are also shown.

\section{Discussion}

The past decade has seen a great success in detecting magnetar outbursts, mainly thanks to the prompt response and monitoring of the Swift mission, and to the dedicated follow-up programs of Chandra, XMM-Newton, and more recently NUSTAR. The detailed study of about 10 outbursts has shown many common characteristics (see [[] for a review), although the precise triggering mechanism of these events, as well as the energy reservoir responsible for sustaining the emission over many months, remain uncertain.

The initial behavior of the 2013 outburst decay of SGR 1745-2900 was compatible with those observed in other magnetars. The outburst peak, the thermal emission peaked at about $1 \mathrm{keV}$, the small radiating surface (about $2 \mathrm{~km}$ in radius) and the overall evolution in the first few months were consistent with the behavior observed for other outbursts. However, after an additional year of X-ray monitoring, it became clear that the subsequent evolution of SGR 1745-2900 showed distinct characteristics. The flux decay appears extremely slow: it is the first time that we observe a magnetar with a quiescent luminosity $<10^{34} \mathrm{erg} \mathrm{s}^{-1}$ remaining at a luminosity $>10^{35} \mathrm{erg} \mathrm{s}^{-1}$ for more than $1 \mathrm{yr}$ and with a temperature decreasing from the initial $\sim 1 \mathrm{keV}$ by less than $10 \%$.

\subsection{Crustal cooling modeling}

In Fig. 田 (left-hand panel, lower curves) we show an example of the expected cooling curve of a magnetar with the same characteristics of SGR 1745-2900 ([ए8]). We assume that (i) a sudden large energy release, $\simeq 10^{45} \mathrm{erg}$, heats up a layer of the outer crust up to $3 \times 10^{9} \mathrm{~K}$; (ii) the event affects the entire magnetar surface; (iii) the layer where the energy is injected extends from an external boundary at $\rho_{\text {OUT }} \sim 3 \times 10^{9} \mathrm{~g} \mathrm{~cm}^{-3}$ to an inner boundary at $\rho_{\text {IN }} \sim 2$ and $4 \times 10^{10}$ $\mathrm{g} \mathrm{cm}^{-3}$ (we show these two cases in the two lower curves of Fig. $\theta$, left-hand panel). Even in this most favorable case, the high luminosities observed at late times are difficult to reconcile with any 

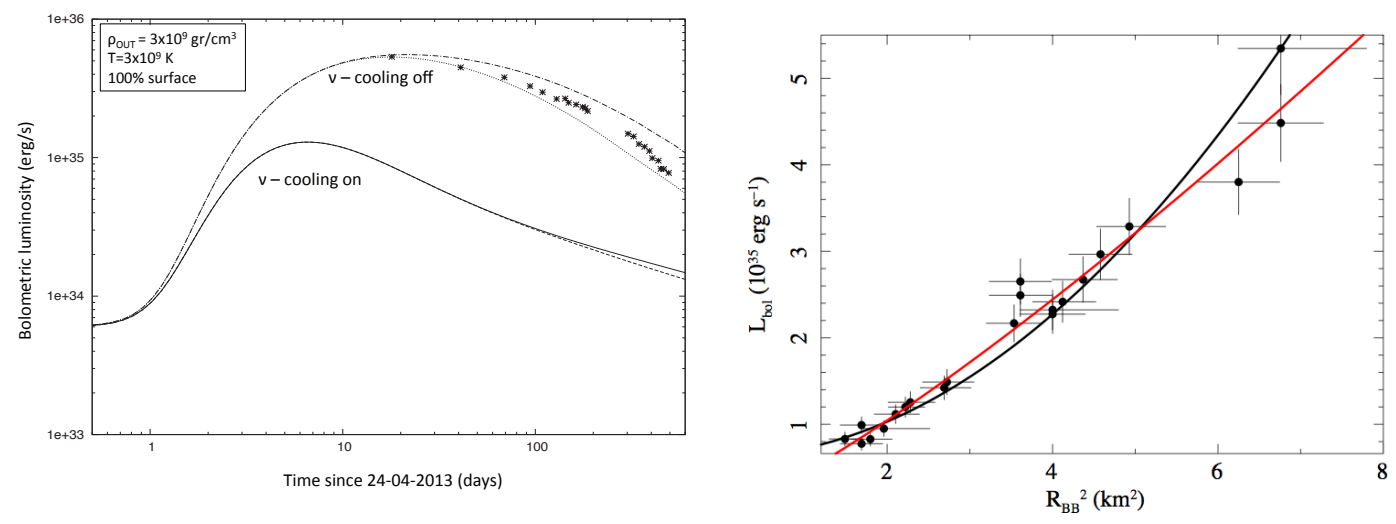

Figure 4: Left-hand panel: crustal cooling curves attempting at modelling the luminosity decrease of SGR 1745-2900. Luminosities are bolometric and calculated assuming a distance of $8.3 \mathrm{kpc}$. For the neutrino-cooling on and off set of curves, the lower and upper curves are relative to $\rho_{\mathrm{IN}}=2$ and $4 \times 10^{10}$ $\mathrm{g} \mathrm{cm}^{-3}$, respectively. Right-hand panel: bolometric luminosity as a function of the square of the blackbody radius at infinity. Solid lines represent the fits with a quadratic function (black) and a generic power law with index $\alpha=1.23(8)$ (red).

cooling model. Injecting more energy or changing $\rho_{\text {OUT }}$ only affects the peak luminosity during the first days or weeks, and injecting energy deeper into the crust (i.e. at higher $\rho_{\mathrm{IN}}$ ) is expected to change the late time evolution only slightly. This can be seen by comparing the solid and dashed lines in the left-hand panel of Fig. 团, which correspond to $\rho_{\mathrm{IN}}=2$ and $4 \times 10^{10} \mathrm{~g} \mathrm{~cm}^{-3}$, respectively.

For illustrative purposes, we also show the cooling curves obtained when plasmon and synchrotron neutrino processes are switched off (see the upper curves in the left-hand panel of Fig. (耳). These provide a much closer match to the data, but there is no clear reason why these neutrino processes should not operate in these conditions. Another possibility to fit the data is to tune the energy injection, which must be maintained during the first $\sim 200 \mathrm{~d}$, resulting in a higher luminosity at late times. If we assume that only a region $5 \mathrm{~km}$ in radius is affected (this is closer to the $\sim 2 \mathrm{~km}$ emitting region observed), we need a continuous injection of at least $\sim 10^{44} \mathrm{erg} \mathrm{s}^{-1} \mathrm{~d}^{-1}$ for about $200 \mathrm{~d}$. However, a physical mechanism that can operate for such a long time-scale is not known. A possibility might be a continuous injection of energy to keep the surface at high temperatures for so long, although in this latter case we should possibly expect more SGR-like bursts during the first hundreds days.

\subsection{Bombardment by magnetospheric currents in a bundle}

A valid alternative model to the crustal cooling scenario invokes the presence of magnetospheric currents flowing along a gradually shrinking magnetic bundle, and heating the surface from outside. In this scenario, a quasi steady-state outflow of electrons and positrons is maintained thanks to magnetic pair production close to the surface. The non-negligible electric voltage along the magnetic field lines and the radiative force due to Compton scattering regulate the streams of positrons and electrons along the field line ([ए]]). 


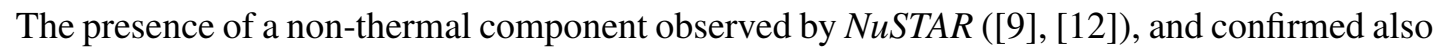
by our XMM-Newton observations on a much longer temporal baseline, is suggestive of a large density of magnetospheric particles which boost thermal photons emitted from the surface via resonant Compton scattering, providing the power law component. In this context, the observed $\sim 2 \mathrm{~km}$ size of the emitting blackbody is consistent with a relatively small twisted magnetic bundle. In the scenario in which the outburst evolution is dominated by an untwisting bundle and the poloidal magnetic field has a dipole geometry, the luminosity is expected to decrease with the square of the blackbody area $\left(A_{\mathrm{BB}}=4 \pi R_{\mathrm{BB}}^{2} ;[[\mathrm{ZO}])\right.$. A flatter dependence may arise from a more complex field geometry. In Fig. 团 we show the fits of the bolometric luminosity as a function of $R_{\mathrm{BB}}^{2}$ with two different models, a quadratic function $L_{\mathrm{bol}} \propto A_{\mathrm{BB}}^{2}$ (black line; $\chi_{v}^{2}=1.3$ for 23 d.o.f.) and a power law $L_{\mathrm{bol}} \propto A_{\mathrm{BB}}^{\alpha}$ (red line; $\chi_{v}^{2}=0.8$ for 23 d.o.f.). For the latter we find $\alpha=1.23(8)$.

\section{Conclusions}

The spectacular angular resolution of Chandra and the large effective area of XMM-Newton allowed us to collect an unprecedented data set covering the outburst of SGR 1745-2900.

We found three different timing solutions between 2013 April 29 and 2014 August 30, which show that the source period derivative has changed at least twice, from $6.6 \times 10^{-12} \mathrm{~s} \mathrm{~s}^{-1}$ in 2013 April at the outburst onset, to $3.3 \times 10^{-11} \mathrm{~s} \mathrm{~s}^{-1}$ in 2014 August. While the first $\dot{P}$ change could be related with the occurrence of an SGR-like burst ([12]), no burst has been detected from the source close in time to the second $\dot{P}$ variation. This further change in the rotational evolution of the source might be related with the timing anomaly observed in the radio band around the end of 2013 ([ए]]), unfortunately during our observing gap.

The $0.3-8 \mathrm{keV}$ source spectrum is perfectly modeled by a single blackbody with temperature cooling from $\sim 0.9$ to $0.75 \mathrm{keV}$ in about $1.5 \mathrm{yr}$. A faint non-thermal component is observed with $X M M-N e w t o n$. It dominates the flux at energies $\gtrsim 8 \mathrm{keV}$ at all the stages of the outburst decay, with a power law photon index ranging from $\sim 1.7$ to $\sim 2.6$. It is most probably due to resonant Compton scattering on to non-relativistic electrons in the magnetosphere.

Crustal cooling models have difficulty in explaining the high luminosity of this outburst and its extremely slow flux decay. If the outburst evolution is indeed due to crustal cooling, then magnetic energy injection needs to be continuous over at least the first $\sim 200 \mathrm{~d}$. The presence of a small twisted bundle sustaining currents bombarding the surface region at the base of the bundle, and keeping the outburst luminosity so high, appears a viable scenario to explain this particular outburst. However, detailed numerical simulations are needed to confirm this possibility.

\section{Acknowledgements}

FCZ is supported by an NWO Vidi Grant (PI: Rea) and the European COST Action MP1304 (NewCOMPSTAR), and acknowledges CSIC-IEEC for kind hospitality during part of the work.

\section{References}

[1] S. Mereghetti, The strongest cosmic magnets: soft gamma-ray repeaters and anomalous $X$-ray pulsars, A\&ARv. 15 (2008) 225 [arXiv: 0804 . 0250]. 
[2] N. Rea \& P. Esposito, Magnetar outbursts: an observational review, in D. F. Torres, N. Rea, eds, Astrophysics and Space Science Proceedings, High-Energy Emission from Pulsars and Their Systems. Springer-Verlag, Berlin (2011) 247 [arXiv: 1101 . 4 472].

[3] N. Rea, et al., A Low-Magnetic-Field Soft Gamma Repeater, Science 330 (2010) 944 [arXiv:1010.2781].

[4] N. Rea, et al., A New Low Magnetic Field Magnetar: The 2011 Outburst of Swift J1822.3-1606, ApJ 754 (2012) 27 [arXiv: 1203.6449$].$

[5] N. Rea, et al., 3XMM J185246.6+003317: Another Low Magnetic Field Magnetar, ApJL 781 (2014) L17 [arXiv:1311.3091].

[6] P. Scholz, et al. Post-outburst X-Ray Flux and Timing Evolution of Swift J1822.3-1606, ApJ 761 (2012) 66 [arXiv: 1204.1034$]$.

[7] A. Tiengo, et al., A variable absorption feature in the X-ray spectrum of a magnetar, Nature $\mathbf{5 0 0}$ (2013) 312 [arXiv: 1308 .4987].

[8] J. A. Kennea, et al., Swift Discovery of a New Soft Gamma Repeater, SGR 1745-2900, near Sagittarius $A^{*}$, ApJ 770 (2013) 24 [arXiv: 1305 . 2128].

[9] K. Mori, et al., NuSTAR Discovery of a 3.76 s Transient Magnetar Near Sagittarius A*, ApJ 770 (2013) 23 [arXiv:1305.1945].

[10] N. Rea, et al., A Strongly Magnetized Pulsar within the Grasp of the Milky Way's Supermassive Black Hole, ApJL 775 (2013) L34 [arXiv: 1307 . 6331].

[11] R. S. Lynch, et al., Green Bank Telescope and Swift X-ray Telescope Observations of the Galactic Center Radio Magnetar SGR 1745-2900, arXiv: 1412.0610.

[12] V. M. Kaspi, et al., Timing and Flux Evolution of the Galactic Center Magnetar SGR 1745-2900, ApJ 786 (2014) 84 [arXiv: 1403.5344 ].

[13] L. Nobili, R. Turolla, S. Zane, X-ray spectra from magnetar candidates - I. Monte Carlo simulations in the non-relativistic regime, MNRAS 386 (2008a) 1527 [arXiv: 0802.2647 ].

[14] L. Nobili, R. Turolla, S. Zane, X-ray spectra from magnetar candidates - II. Resonant cross-sections for electron-photon scattering in the relativistic regime, MNRAS 389 (2008b) 989 [arXiv:0806.3714].

[15] S. Zane, N. Rea, R. Turolla, L. Nobili, X-ray spectra from magnetar candidates - III. Fitting SGR/AXP soft X-ray emission with non-relativistic Monte Carlo models, MNRAS 398 (2009) 1403 [arXiv:0906.1135].

[16] C. Thompson, M. Lyutikov, S. R. Kulkarni, Electrodynamics of Magnetars: Implications for the Persistent X-Ray Emission and Spin-down of the Soft Gamma Repeaters and Anomalous X-Ray Pulsars, ApJ 574 (2002) 332 [arXiv: astro-ph/ 0110677 ].

[17] G. Ponti, et al., in D. F. Torres, O. Reimer, eds, Astrophysics and Space Science Proceedings, Cosmic Rays in Star-Forming Environments. Springer-Verlag, Berlin 34 (2013) 331 [arXiv: 1210 . 3034 ].

[18] J. A. Pons \& N. Rea, Modeling Magnetar Outbursts: Flux Enhancements and the Connection with Short Bursts and Glitches, ApJL $\mathbf{7 5 0}$ (2012) L6 [arXiv: 1203 . 4506].

[19] A. M. Beloborodov, Electron-Positron Flows around Magnetars, ApJ 777 (2013) 114 [arXiv:1209.4063].

[20] A. M. Beloborodov, Untwisting Magnetospheres of Neutron Stars, ApJ 703 (2009) 1044 [arXiv:0812.4873]. 